\title{
Accurate FIC-FEM formulation for the multidimensional steady-state advection-diffusion-absorption equation
}

\author{
Eugenio Oñate ${ }^{\mathrm{a}, \mathrm{b}, *}$, Prashanth Nadukandii ${ }^{\mathrm{a}, \mathrm{c}}$, Juan Miquel ${ }^{\mathrm{a}, \mathrm{b}}$ \\ ${ }^{a}$ Centre Internacional de Metodes Numerics a l'Enginyeria (CIMNE), Barcelona, Spain \\ ${ }^{\mathrm{b}}$ Universitat Politècnica de Catalunya (UPC), Spain \\ ${ }^{\mathrm{c}}$ School of Mathematics, The University of Manchester, Oxford Rd, Manchester, UK
}

Available online xxxxx

\section{Highlights}

- Accurate stabilized FIC-FEM formulation for the multidimensional steady-state advection-diffusion-absorption equation.

- Accurate FEM solutions with linear elements using two stabilization parameters.

\begin{abstract}
In this paper we present an accurate stabilized FIC-FEM formulation for the multidimensional steady-state advection-diffusionabsorption equation.

The stabilized formulation is based on the Galerkin FEM solution of the governing differential equations derived via the Finite Increment Calculus (FIC) method using two stabilization parameters. The value of the two stabilization parameters ensuring an accurate nodal FEM solution using uniform meshes of linear elements is obtained from the optimal values for the 1D problem.

The accuracy of the new FIC-FEM formulation is demonstrated in the solution of 2D steady-state advection-diffusionabsorption problems for a range of physical parameters and boundary conditions.
\end{abstract}

(C) 2017 Elsevier B.V. All rights reserved.

Keywords: Advection-diffusion-absorption; Finite element method; Finite increment calculus

\section{Introduction}

It is well known that the numerical solution of the advection-diffusion-absorption problem by the standard Galerkin FEM is prone to exhibit global, Gibbs and dispersive oscillations [1-3]. The solution of the stationary problem by the FEM exhibits spurious global oscillations for the convection-dominated case. The local Gibbs oscillations are exhibited along the characteristic layers for the 2D/3D convection-dominated case. For the absorptiondominated cases Gibbs oscillations may be found near the Dirichlet boundaries and in the regions where the distributed

Dedicated to J. Tinsley Oden on its 80th birthday.

* Correspondence to: CIMNE, 08034, Universitat Politècnica de Catalunya (UPC), Spain.

E-mail addresses: onate@cimne.upc.edu, majesus@cimne.upc.edu (E. Oñate). 
source term is nonregular. The solution of the transient problem may exhibit dispersive oscillations when the initial solution and/or the distributed source term are nonregular.

Control over the global instability has been achieved via the streamline-upwind Petrov-Galerkin (SUPG) [4-6], Taylor-Galerkin [7], characteristic Galerkin [8,9], Galerkin least squares (GLS) [10], bubble functions [11-13], variational multiscale (VMS) [14], characteristic-based split (CBS) [15] and finite increment calculus (also known as finite calculus) (FIC) based methods [16-21]. A thorough comparison of some of these methods can be found in [22]. Oñate and Manzan [17,18] showed that many of the above stabilized methods can be recovered using the FIC equations via an appropriate definition of the stabilization parameters. Nevertheless nonregular solutions continue to exhibit the Gibbs and dispersive oscillations.

Several shock-capturing nonlinear Petrov-Galerkin methods were proposed to control the Gibbs oscillations observed across characteristic internal/boundary layers for the convection-diffusion problem [19,23-29]. A state of the art review of these and several other shock-capturing methods for the convection-diffusion equations was done in [30]. Reactive terms were not considered in the design of these methods and hence they fail to control the localized oscillations in the presence of these terms. Exceptions to this are the consistent approximate upwind (CAU) method [23], the methods presented in [31] and those that take the CAU method as the starting point [26]. Nevertheless, the expressions for the stabilization parameters therein were never optimized for reactive instability and often the solutions are over-diffusive in these cases.

In the quest to gain reactive stability several methods were built upon the existing frameworks of methods that control global oscillations. Extension of the SUPG method were proposed for the convection-diffusion-reaction problem, viz. the DRD [32] and (SU+C)PG [33] methods. Based on the GLS method, linear stabilized methods were proposed, viz. the GGLS method [34] for the diffusion-reaction problem and the GLSGLS method [35] for the convection-diffusion-production problem. Within the framework of stabilization via bubbles we note the USFEM method [36] for the diffusion-reaction problem, the improved USFEM method [37] and the link cutting bubbles procedure [38] for the convection-diffusion-reaction problem. Based on the VMS method linear stabilized methods were proposed for the convection-diffusion-reaction problem, viz. the ASGS method [39], the SGS-GSGS method [40] and others [41,42]. Using the FIC equations a nonlinear method based on a single stabilization parameter was proposed for the convection-diffusion-absorption problem in 1D [20] and higher dimensions [21]. Nodally exact Ritz discretizations of the 1D diffusion-reaction equation by a variational FIC method using a single stabilization parameter were presented in [43].

Control over the dispersive oscillations for the transient convection-diffusion problem via linear Petrov-Galerkin methods were discussed in [44] and using space-time finite elements in [45]. As for the linear methods, optimizing the expressions of the stabilization parameters to attain monotonicity will lead to solutions that are at most first-order accurate.

Generally the homogeneous steady convection-diffusion-reaction problem in 1D has two fundamental solutions. Thus in principle one can use two stabilization parameters for designing linear stabilized methods that are nodally exact in 1D. Following this line several "two-parameter methods" were designed to be nodally exact for the stationary problem in $1 \mathrm{D}[35,38,42]$.

In [46,47] we presented a FIC-based nonlinear high-resolution Petrov-Galerkin (HRPG) method for convectiondiffusion-absorption problems. The method has two parameters and is capable of reproducing high-resolution numerical solutions for both the stationary and transient regimes.

Following these lines, Oñate et al. [48] presented an accurate FIC-FEM formulation for the 1D steady-state and transient advection-diffusion-absorption equation in the exponential and propagation regimes using two stabilization parameters. The FIC-FEM formulation provided excellent results for both regular and irregular meshes.

In this work we extend to the multidimensional steady-state case the 1D FIC-FEM formulation presented in [48]. Due to the particular features of the multidimensional Helmholtz problem, in this work we will only consider the advection-diffusion-absorption situations. The optimal value of two stabilization parameters ensuring a quasi-exact (nodal) FEM solution using uniform meshes of linear 2-noded elements is obtained as an extension of the analogous expressions for the 1D problem derived in [48]. In the absence of the absorption term the formulation simplifies to the standard one-parameter Petrov-Galerkin approach for the advection-diffusion problem. For the diffusion-absorption case one stabilization parameter is just needed and the diffusion-type stabilization term is identical to that obtained by Felippa and Oñate [43] using a variational FIC approach.

The lay-out of the paper is the following. In the next section we formulate the FIC form of the equations governing steady-state multidimensional convection-diffusion-absorption problem. The finite element discretization 
using linear elements is presented. Then the optimal stabilization parameters yielding quasi-exact nodally solutions for the sourceless case are obtained. The accuracy of the multidimensional FIC-FEM formulation is verified in the solution of a number of steady-state advection-diffusion-absorption problems in the exponential and propagation regimes using uniform and irregular meshes of 3-noded triangles and 4-noded quadrilateral elements. Accurate solutions are obtained in all cases. Quasi-exact solutions are obtained when regular meshes are used, as expected.

\section{The multidimensional steady-state advection-diffusion-absorption problem}

\subsection{Governing equations}

Transport balance

$$
\begin{aligned}
& r_{s}=0 \quad \text { in } \Omega \\
& r_{s}:=\rho c \mathbf{v}^{T} \nabla \boldsymbol{\phi}-\nabla^{T} \mathbf{D} \nabla \phi+s \phi-Q
\end{aligned}
$$

where $\rho$ and $c$ are the density and the heat ...

For 3D problems,

$$
\mathbf{v}=\left[v_{1}, v_{2}, v_{3}\right] T \quad, \quad \mathbf{D}=\left[\begin{array}{ccc}
k_{1} & 0 & 0 \\
0 & k_{2} & 0 \\
0 & 0 & k_{3}
\end{array}\right] \quad, \quad \nabla=\left[\frac{\partial}{\partial x_{1}}, \frac{\partial}{\partial x_{2}}, \frac{\partial}{\partial x_{3}}\right]^{T}
$$

In Eqs. (1)-(2b) $\phi$ is the transported variable (for instance, the temperature in a heat transfer problem), $u$ is the velocity, $\rho, c$ and $k$ are the density, the specific flux parameter and the conductivity of the material, respectively, $s$ is the reaction parameter ( $s>0$ is the absorption or dissipation parameter and $s<0$ is the production parameter). In the following, and unless otherwise specified, we will assume that the problem parameters $(u, \rho, c, k, s)$ are constant over the analysis domain.

\section{Boundary conditions}

$$
\begin{aligned}
& \phi-\phi^{n}=0 \quad \text { on } \Gamma_{\phi} \\
& r_{\Gamma}=0 \quad \text { on } \Gamma_{q}
\end{aligned}
$$

with

$$
\begin{aligned}
& r_{\Gamma}:=-q_{n}+q_{n}^{p} \\
& q_{n}=\mathbf{q}^{T} \mathbf{n} \quad, \quad \mathbf{q}=\mathbf{v} \phi-\mathbf{D} \nabla \phi
\end{aligned}
$$

where $\mathbf{n}$ is the unit vector normal to the boundary.

In Eqs. (42)-(6) $\phi^{p}$ and $q^{p}$ are the prescribed values of the transported variable and the outgoing flux at the Dirichlet and Neumann boundaries $\Gamma_{\phi}$ and $\Gamma_{q}$, respectively, with $\Gamma_{\phi} \cup \Gamma_{q}=\Gamma, \Gamma$ being the total boundary of the domain

In this work we will consider cases for which $s \geq 0$ only. This includes the following particular problems:

(i) Advection-diffusion-absorption $(|\mathbf{v}| \neq 0, K \neq 0, s>0)$.

(ii) Advection-diffusion $(|\mathbf{v}| \neq 0, K \neq 0, s=0)$. 
(iii) Diffusion-absorption $(|\mathbf{v}|=0, K \neq 0, s>0)$.

(iv) Advection-absorption $(|\mathbf{v}| \neq 0, K=0, s>0)$.

In the above $K$ is the average diffusion given by $K=\left[\frac{1}{n_{d}} \sum_{i=1}^{n_{d}} k_{i}\right]^{1 / 2}$, where $n_{d}$ is the number of space dimensions (i.e. $n_{d}=2$ for $2 \mathrm{D}$ problems).

\subsection{Finite increment calculus (FIC) expressions}

\section{Transport balance}

$$
r_{s}-\underline{\frac{1}{2} \mathbf{h}^{T} \nabla r_{s}}=0
$$

Boundary conditions

$$
\begin{aligned}
& \phi-\phi^{p}=0 \quad \text { on } \Gamma_{\phi} \\
& r_{\Gamma}-\underline{\frac{1}{2} h_{n} r_{s}}=0 \quad \text { on } \Gamma_{q} \quad \text { with } h_{n}=\mathbf{h}^{T} \mathbf{n}
\end{aligned}
$$

Eqs. (7) and (8b) are obtained by expressing the balance of fluxes in an arbitrary segment of finite length $h$ (termed the characteristic length) within the problem domain and at the Neumann boundary, respectively. The variations of the transported variable within the balance segment are approximated by Taylor series expansions retaining one order higher terms than in the infinitesimal theory [16]. The underlined terms in Eqs. (7) and (8) emanate from these series expansions and they lead naturally to stabilized numerical schemes.

Note that $r_{s} \neq 0$ and $r_{\Gamma} \neq 0$ in the FIC balance equations (7) and (8). However, as the characteristic length $h$ tends to zero the FIC differential equations gradually recover the standard infinitesimal form, giving in the limit (for $h=0$ ) $r_{s}=0$ in $\Omega$ and $r_{\Gamma}=0$ on $\Gamma_{q}$.

As in all stabilized methods, the stability and accuracy of the numerical solution depends on the values of the stabilization parameter, i.e. of the characteristic length vector $\mathbf{h}$. At the discrete level the length of $\mathbf{h}$ can be related to the characteristic dimension of the (macroscopic) domain within which the space derivatives are computed. At the discretization level it is usual to express the length of $\mathbf{h}$ as a proportion of a typical grid dimension (i.e. the element length for 1D FEM problems or a characteristic element dimension for 2D and 3D problems) [16].

The FIC governing equations lead to stabilized numerical schemes using whatever numerical method. It is interesting that many of the standard stabilized FEM can be recovered using the FIC-FEM formulation. The FICFEM method has been successfully applied to the finite element solution problems of convection-diffusion [16-19], diffusion-absorption and Helmholtz [43], advection-diffusion-absorption [20,21], advection-diffusion-reaction [48], incompressible fluid flow [49-53], fluid-structure-interaction [54-56], particulate flows and standard and incompressible solid mechanics [57-59]. Applications of the FIC stabilization approach to meshless problems solved using the finite point method are reported in [60-63].

\subsection{The $1 D$ advection-diffusion-absorption problem}

The FIC-FEM equations for the 1D steady-state advection-reaction-absorption problems are [48]

\section{Transport balance}

$$
\begin{aligned}
& r_{s}-\frac{1}{2} h \frac{d r_{s}}{d x}=0 \quad \text { in } \Omega \\
& r_{s}:=\rho c v \frac{d \phi}{d x}-\frac{d}{d x}\left(k \frac{d \phi}{d x}\right)+s \phi-Q
\end{aligned}
$$




\section{Boundary conditions}

$$
\begin{aligned}
& \phi-\phi^{p}=0 \quad \text { on } \Gamma_{\phi} \\
& r_{\Gamma}+\frac{h}{2} r_{s}=0 \quad \text { on } \Gamma_{q}
\end{aligned}
$$

with

$$
r_{\Gamma}=\left(-\rho c u \phi+k \frac{d \phi}{d x}\right) n+q^{p}=0
$$

when $M$ defines the sign of the normal direction at the boundary edges.

For simplicity we will assume that $\Gamma_{\phi}$ and $\Gamma_{q}$ are placed at $x=0$ and $x=l$, respectively. Hence, $n=+1$ at $x=l$.

Oñate et al. [48] have proposed the following expression for the characteristic length parameter

$$
h=h_{v}+h_{r} \quad \text { with } h_{v}=\alpha_{v} l^{e} \quad, \quad h_{r}=2 \alpha_{r} \frac{k}{r} \frac{d \phi}{d x}
$$

where $l^{e}$ is the element length and $\alpha_{v}$ and $\alpha_{r}$ are stabilization parameters. Indices $v$ and $r$ refer to the velocity and reaction contributions to the characteristic length parameter.

Application of the standard Galerkin FEM to the FIC equations (9)-(11) leads to the following system of discretized equations

$$
[\mathbf{K}+\mathbf{C}+\mathbf{S}] \boldsymbol{\phi}=\mathbf{f}
$$

where vector $\boldsymbol{\phi}=\left[\phi_{1}, \phi_{2}, \ldots, \phi_{N}\right]^{T}$ lists the values of the approximated transported variable at the $N$ nodes in the mesh, $\mathbf{K}$ is the diffusion matrix, $\mathbf{C}$ is the convection matrix, $\mathbf{S}$ is the radiation matrix and $\mathbf{f}$ is the nodal external flux vector. These matrices and vector $\mathbf{f}$ are assembled from the element contributions given by

$$
\begin{aligned}
K_{i j}^{e} & =\int_{l^{e}}\left[\frac{d N_{i}}{d x} \bar{k} \frac{d N_{j}}{d x}-\frac{d N_{i}}{d x} \frac{h_{v}}{2} \frac{d}{d x}\left(k \frac{d N_{j}}{d x}\right)\right] d x \\
C_{i j}^{e} & =-\int_{l^{e}} \frac{d N_{i}}{d x} \rho c \bar{v} N_{j} d x \quad, \quad S_{i j}^{e}=\int_{l^{e}} s N_{i} N_{j} d x \\
f_{i}^{e} & =\int_{l^{e}}\left[N_{i}+\alpha_{r} \frac{l^{e}}{2} \frac{d N_{i}}{d x}\right] Q d x
\end{aligned}
$$

Note that the second term in $K_{i j}^{e}$ vanishes for the linear 2-noded elements.

The expression for the equivalent diffusion $\bar{k}$ in $K_{i j}^{e}$ (Eq. (16a)) is given by

$$
\bar{k}=k+k_{v}+k_{r}
$$

with $k_{v}=\alpha_{v} \frac{\rho c v l^{e}}{2}=\alpha_{v} \gamma k$ and $k_{r}=\alpha_{r} k$, where $\gamma=\frac{v l^{e}}{2 k}$ is the element Peclet number.

On the other hand, the equivalent velocity $\bar{v}$ in matrix $\mathbf{C}$ (Eq. (16b)) is given by

$$
\bar{v}=\left(1-\frac{\alpha_{v}}{2} \sigma\right) v
$$

where $\sigma=\frac{w}{2 \gamma}=\frac{s l^{e}}{\rho c v}$ is the Damköhler number.

The above expressions show that the definition of the characteristic length parameter of Eq. (14) leads to a linear problem with an equivalent diffusion $\bar{k}$ and an equivalent velocity $\bar{v}$.

In [48] it is shown that exact nodal solutions for a uniform mesh of 2-noded elements are found using the following expressions for the stabilization parameters $\alpha_{v}$ and $\alpha_{r}$ :

$$
\begin{aligned}
& \alpha_{v}=\frac{2}{\sigma}\left(1-\frac{\sigma \tanh \gamma}{\xi-1}\right) \\
& \alpha_{r}=\gamma\left[\frac{\sigma}{3}\left(\frac{\xi+2}{\xi-1}\right)-\alpha_{v}\right]-1
\end{aligned}
$$

with $\xi=\frac{\cosh \lambda}{\cosh \gamma}$ with $\lambda=\left(\gamma^{2}+w\right)^{1 / 2}$. 
Examples of the accuracy of the FIC-FEM formulation for 1D advection-diffusion-reaction problems (including Helmholtz problems) using uniform and non-uniform meshes of 2-noded elements are given in [48]. Exact nodal solution for uniform meshes are obtained in all cases as expected.

Remark 1. Equivalent expressions of the "exact" stabilization parameters $\alpha_{v}$ and $\alpha_{r}$ in terms of $\gamma$ and $w$ are given in [48]. Note that $\alpha_{r}$ is termed $\alpha_{g}$ in [48]. Also in the expression of $\alpha_{r}$ in Eq. (19b) of [48] the -1 term is missing. The correct expression is given in Eq. (19b).

The above FIC-FEM formulation for the steady-state 1D advection-diffusion-reaction problem will be extended for solving the multidimensional advection-diffusion-absorption problem as presented next.

\section{FIC-FEM formulation for the multidimensional steady-state advection-diffusion-absorption problem}

\subsection{Definition of the characteristic length vector}

The characteristic length vector $\mathbf{h}$ is designed so that the expression for all matrices and vectors reduce to those given for the $1 \mathrm{D}$ case in the previous section.

With this objective in mind the following expression for the characteristic length vector has been chosen

$$
\mathbf{h}=\mathbf{h}_{v}+\mathbf{h}_{r}+\mathbf{h}_{s c}
$$

with $\mathbf{h}_{v}$ is a length vector along the velocity direction defined as

$$
\mathbf{h}_{v}=\alpha_{v} l_{v} \frac{\mathbf{v}}{|\mathbf{v}|}
$$

$\mathbf{h}_{r}$ is a length vector induced by the radiation (absorption) effects,

$$
\mathbf{h}_{r}=\mathbf{H}_{r} \nabla \phi \quad \text { with } \quad \mathbf{H}_{r}=\frac{2}{r_{s}}\left[\mathbf{D}_{s}+\alpha_{r} D \hat{\mathbf{v}} \hat{\mathbf{v}}^{T}\right]
$$

where $\hat{\mathbf{v}}$ is a unit velocity vector (i.e. $\hat{\mathbf{v}}=\frac{\mathbf{v}}{|\mathbf{v}|}$ ) and

$$
D=\hat{\mathbf{v}}^{T} \mathbf{D} \hat{\mathbf{v}}
$$

Matrix $\mathbf{D}_{s}$ is defined for different element types as follows.

\section{3-noded triangles and 4-noded tetrahedra:}

$$
\mathbf{D}_{s}=\frac{s}{(n+1)} \sum_{i=1}^{n} \mathbf{l}_{i} \mathbf{l}_{i}^{T}
$$

where $\mathbf{l}_{i}$ is the vector joining the barycentre of the element and the $i$ th node and $n$ is the number of nodes of the element.

Any other element: $\mathbf{D}_{s}=[0]$

Finally, in Eq. (20) $\mathbf{h}_{s c}$ is a shock-capturing length vector in the direction of the gradient of the solution. This vector accounts for the Gibbs oscillations across characteristic internal/boundary layers for convection-diffusion problems. It is defined as

$$
\mathbf{h}_{s c}=h_{s c} \widehat{\nabla \phi}
$$

where $\widehat{\nabla \phi}=\frac{\nabla \phi}{|\nabla \phi|}$ is the unit gradient vector, and

$$
h_{s c}=\left(1-\beta^{2}\right)\left[l_{s c} \operatorname{sgn}\left(r_{s}\right)-\frac{2|\nabla \phi|}{r_{s}}\left(\mathbf{D}+\mathbf{D}_{s}\right):\left(\mathbf{I}-\hat{\mathbf{v}} \hat{\mathbf{v}}^{T}\right)\right]
$$


In (24b) $\beta$ is a parameter that depends on the angle $\theta$ between the velocity vector and the gradient vector. For 3-noded triangular elements it is defined as

$$
\beta=\left\{\begin{array}{lll}
1 & \text { if } \quad \theta<\theta_{c} \\
\hat{\mathbf{v}}^{T} \widehat{\nabla \phi} & \text { if } \quad \theta \geq \theta_{c}
\end{array}\right.
$$

where $\theta_{c}$ is a critical angle. In our work we have taken $\theta_{c}=20^{\circ}$.

The parameter $\beta$ controls the amount of shock-capturing nonlinear diffusion active at any point of the domain. When the gradient vector is parallel to the velocity vector then $\beta=1, h_{s c}$ vanishes and the linear stabilization terms suffice to diminish spurious numerical oscillations about the layers. In this case the solution is essentially 1D and the linear stabilization terms are a direct extension to $2 \mathrm{D}$ of the optimal $1 \mathrm{D}$ terms. The expression $\beta=\widehat{\mathbf{v}} \cdot \widehat{\nabla \phi}$ takes a unit value in the aforesaid case and becomes zero when the layer gradient is orthogonal to the velocity. Multiplying the shock-capturing term with $1-\beta^{2}$ gradually increases its magnitude from zero, when the gradient vector is aligned to velocity vector, to a maximum value when the layer gradient is orthogonal to the velocity. However, we have found this model for $\beta$ not effective near domain corners and in the vicinity of discontinuous boundary data where the layer gradient may have a spurious direction. For quadrilateral meshes we have found the following expression for $\beta$ to be more effective

$$
\beta=\left(1-\frac{\operatorname{std}(\widehat{\mathbf{v}} \cdot \widehat{\nabla \phi})}{\max (\operatorname{std}(\widehat{\mathbf{v}} \cdot \widehat{\nabla \phi}))}\right)(\widehat{\mathbf{v}} \cdot \widehat{\nabla \phi})
$$

where, the std() operator returns the standard deviation of its argument evaluated at the Gauss points and the $\max ()$ operator returns the maximum value of its argument over all elements. The rationale for this model is that Q1 elements which have spurious layer gradients will also have a larger standard deviation in the values of $\widehat{\mathbf{v}} \cdot \widehat{\nabla \phi}$. The higher the standard deviation the closer is $\beta$ to zero and the higher is the magnitude of the shock-capturing stabilization term. For P1 elements $\widehat{\mathbf{v}} \cdot \widehat{\nabla \phi}$ is constant within an element and hence the standard deviation is zero. So Eq. (25) reduces to $\beta=\widehat{\mathbf{v}} \cdot \widehat{\nabla \phi}$ and results in no additional benefits as obtained for the Q1 elements. Another possibility is to take the standard deviation of $\widehat{\mathbf{v}} \cdot \widehat{\nabla \phi}$ in a patch of elements surrounding the chosen element. However, it adversely affected the convergence of the iterations resulting from the linearization of the shock-capturing terms. Hence for P1 elements the model $\beta=\widehat{\mathbf{v}} \cdot \widehat{\nabla \phi}$ was chosen.

Remark. Matrix $\mathbf{D}_{s}$ of Eq. (23) has the following property

$$
\mathbf{K}_{s}^{e}=\int_{\Omega^{e}}\left(\nabla N_{i}\right)^{T} \mathbf{D}_{s} \nabla N_{j} d \Omega=\left(\mathbf{M}_{L}^{e}-\mathbf{M}_{C}^{e}\right)
$$

where

$$
\mathbf{M}_{C_{i j}}^{e}=s \int_{\Omega^{e}} N_{i} N_{j} d \Omega
$$

and $\mathbf{M}_{L}^{e}$ is the lumped form of $\mathbf{M}_{C}^{e}$. We point out that $\mathbf{K}_{s}^{e}$ is the limit stabilizing diffusion matrix for the diffusionradiation problem for the case of zero diffusion. The diffusion introduced by $\mathbf{D}_{s}$ takes care of the instabilities induced by the irregularity of the triangular mesh near boundaries that develop parabolic layers.

\subsection{Definition of the stabilization parameters}

The stabilization parameters $\alpha_{v}$ and $\alpha_{r}$ in Eqs. (21) and (22a) are given by

$$
\alpha_{v}= \begin{cases}\frac{2}{\sigma_{v}}\left(1-\frac{\sigma_{v} \tanh \gamma_{v}}{\xi-1}\right) & , \quad \sigma \geq 2^{-12} \\ \frac{\sigma_{v}}{3}+\bar{\alpha}_{v}\left(1-\frac{\sigma_{v}}{\gamma_{v}}\right) & , \quad \sigma<2^{-12}\end{cases}
$$

with

$$
\bar{\alpha}_{v}=\operatorname{coth} \gamma_{v}-\frac{1}{\gamma_{v}}
$$


and

$$
\alpha_{r}=\gamma_{v}\left[\frac{\sigma_{v}}{\varphi}\left(\frac{\xi_{v}-1+\varphi}{\xi_{v}-1}\right)-\alpha_{v}\right]-1-\frac{1}{D} \hat{\mathbf{v}}^{T} \mathbf{D}_{s} \hat{\mathbf{v}}
$$

In the above expressions

$$
\begin{aligned}
& \xi_{v}=\frac{\cosh \lambda_{v}}{\cosh \gamma_{v}} \quad \text { with } \quad \lambda_{v}=\left(\gamma_{v}^{2}+w_{v}^{2}\right)^{1 / 2} \\
& \gamma_{v}=\frac{|\mathbf{v}| l_{v}}{2 D} \quad, \quad w_{v}=\frac{s\left(l_{v}\right)^{2}}{D} \quad, \quad \sigma_{v}=\frac{s l_{v}}{|\mathbf{v}|}=\frac{w_{v}}{2 \gamma_{v}}
\end{aligned}
$$

The index $v$ in $\gamma, w$ and $\sigma$ is chosen to distinguish these expressions from their 1D counter parts [48].

In Eq. (28) $\varphi$ is a constant such that $2 \leq \varphi \leq 3$. The "exact" expression of $\alpha_{r}$ for $1 \mathrm{D}$ problems requires choosing $\varphi=3$ (see Eq. (19b)). In our computations for 2D and 3D problems we have obtained good results using $\varphi=2$.

For the definition of the lengths $l_{v}$ and $l_{s c}$ we have chosen

$$
l_{v}=l_{s c}=\sqrt{2 \Omega^{e}}
$$

where $\Omega^{e}$ is the element area.

Remark. The regularized expression of $\alpha_{v}$ in Eq. (26) ensures a good numerical behaviour for a precision of up to

11 digits in the exact value of $\alpha_{v}$.

\section{Advection-diffusion problems $(s=0)$}

The stabilization parameters $\alpha_{v}$ and $\alpha_{r}$ for a zero reaction term are obtained as

$$
\begin{aligned}
& \lim _{w \rightarrow 0} \alpha_{v}\left(\gamma_{v}, w\right)=\alpha_{v}\left(\gamma_{v}\right)=\operatorname{coth} \gamma_{v}-\frac{1}{\gamma_{v}} \\
& \lim _{w \rightarrow 0} \alpha_{r}\left(\gamma_{r}, w\right)=\alpha_{r}(\gamma)=0
\end{aligned}
$$

Diffusion-absorption problems $(|\mathbf{v}|=0)$

The stabilization parameters for a diffusion-absorption term are

$$
\begin{aligned}
& \lim _{\gamma \rightarrow 0} \gamma_{v}\left(\gamma_{v}, w\right)=\alpha_{v}(w)=0 \\
& \lim _{\gamma \rightarrow 0} \gamma_{r}\left(\gamma_{r}, w\right)=\alpha_{r}(w)=\frac{w}{4 \sinh ^{2}\left(\frac{\sqrt{w}}{2}\right)}+\frac{w}{6}-1=\bar{\alpha}_{g}(w) w
\end{aligned}
$$

with

$$
\bar{\alpha}_{g}(w)=\frac{w}{4 \sinh ^{2}\left(\frac{\sqrt{w}}{2}\right)}+\frac{1}{6}-\frac{1}{w}
$$

It can be shown that

$$
\lim _{w \rightarrow 0} \bar{\alpha}_{r}(w)=\frac{1}{12} \quad, \quad \lim _{w \rightarrow 0} \alpha_{r}(w)=\frac{1}{6}
$$

The expression of $\bar{\alpha}_{r}$ for the diffusion limit of Eq. (31b) was derived by Felippa and Oñate [43] using a variational FIC formulation. This expression is identical to that obtained in [48] for the 1D problem [].

Advection-absorption problems $(|K|=0)$

$$
\lim _{\gamma \rightarrow \infty} \gamma_{v}(\gamma, w)=1 \quad, \quad \lim _{\gamma \rightarrow \infty} \alpha_{r}(w)=\alpha_{r}(w) d=\frac{s l_{v}^{2}}{6}
$$

For $|K|=0$ then $\gamma \rightarrow \infty$. In this case it is more appropriate to define $\hat{w}=w D=s l_{v}^{2}$. The stabilization parameters for this case are

$$
\lim _{\gamma \rightarrow \infty} \alpha_{v}(\gamma, \hat{w})=1 \quad, \quad \lim _{\gamma \rightarrow \infty} \alpha_{r}(\gamma, \hat{w})=\alpha_{v}(\hat{w})=\frac{\hat{w}}{6}
$$




\subsection{Finite element discretization}

The weighted residual form of the FIC governing equations (7) and (8) is written as

$$
\int W\left(r_{s}-\frac{1}{2} \mathbf{h}^{T} \nabla r_{s}\right) d \Omega+\int_{\Gamma_{q}} W\left(-q_{n}+q_{n}^{p}-\frac{1}{2} h_{n} r_{s}\right) d \Gamma=0
$$

where $W$ are weighting functions.

Integrating by parts the FIC term in the first integral of (36) gives

$$
\int_{\Omega}\left(W r_{s}+\frac{1}{2}\left(\nabla^{T} W\right) \mathbf{h} r_{s}\right) d \Omega-\oint_{\Gamma_{q}} W\left(-q_{n}+q_{n}^{p}\right) d \Gamma=0
$$

Note that the FIC term has vanished from the boundary integral, as it usual in the FIC-FEM approach $[16,64]$.

Let us substitute the expression for the characteristic vector $\mathbf{h}$ of Eq. (20) into Eq. (37) this gives

$$
\int_{\Omega}\left[W r_{s}+\frac{1}{2}\left(\nabla^{T} W\right)\left(\alpha_{v} l_{v} \hat{\mathbf{v}}+\mathbf{H}_{r} \nabla \phi+h_{s c} \frac{\nabla \phi}{|\nabla \phi|}\right)\right] r_{s} d \Omega+\oint_{\Gamma_{q}} W\left(-q_{n}+q_{n}^{p}\right) d \Gamma=0
$$

The final step is the integration by parts of the advective and diffusive terms in the expression of $r_{s}$ in the first term of the first integral in Eq. (38). This gives, after grouping some terms, the following expression for the weak variational form of the FIC governing equations

$$
\begin{aligned}
& \int_{\Omega}\left[-\left(\nabla^{T} W\right) \overline{\mathbf{v}} \phi+\left(\nabla^{T} W\right) \mathbf{D}_{T} \nabla \phi+W \phi\right] d \Omega+\int_{\Omega}\left(\nabla^{T} W\right) \mathbf{h} \\
& {\left[-\nabla^{T}(\mathbf{D} \nabla \phi)-Q\right] d \Omega=-\int_{\Omega} W Q d \Omega+\oint_{\Gamma_{q}} W q_{n}^{p} d \Gamma=0}
\end{aligned}
$$

where the equivalent velocity $\overline{\mathbf{v}}$ is given by

$$
\overline{\mathbf{v}}=\mathbf{v}+\frac{s}{2} \mathbf{h}_{v}
$$

The expression of the total diffusivity matrix $\mathbf{D}_{T}$ is

$$
\mathbf{D}_{T}=\mathbf{D}+\alpha_{v} \mathbf{D}_{v}+\mathbf{D}_{s}+\alpha_{r} D \hat{\mathbf{v}} \hat{\mathbf{v}}^{T}+\mathbf{D}_{s c} \mathbf{I}
$$

where $\mathbf{D}$ and $\mathbf{D}_{s}$ are defined in Eqs. 2(b) and (23), respectively, $\mathbf{I}$ is the unit matrix and

$$
\begin{aligned}
\mathbf{D}_{v} & =\frac{l_{v}}{2} \hat{\mathbf{v}} \mathbf{v}^{T} \\
\mathbf{D}_{s c} & =\left(\frac{1}{2} l_{s_{c}} \frac{\left|r_{s}\right|}{|\nabla \phi|}-\left(\mathbf{D}+\mathbf{D}_{s}\right):\left(\mathbf{I}-\hat{\mathbf{v}} \hat{\mathbf{v}}^{T}\right)\right)\left(1-\beta^{2}\right)
\end{aligned}
$$

We interpolate the transported variable $\phi$ in the standard FEM fashion over a mesh of elements with $n$ nodes as

$$
\phi \simeq \hat{\phi}=\sum_{i=1}^{n} N_{i} \phi_{i}
$$

where $N_{i}$ are the space shape functions and $\phi_{i}$ the nodal variables.

Introducing Eq. (44) into (39) and using a Galerkin approach $\left(W_{i}=N_{i}\right)$ gives the final system of discretized equations as

$$
[\mathbf{K}+\mathbf{C}+\mathbf{S}] \phi=\mathbf{f}
$$

where $\phi=\left[\phi_{1}, \phi_{2}, \ldots, \phi_{N}\right]^{T}$ is the vector of nodal unknowns, with $N$ being total number of elements in the mesh. 
The rest of matrices and vector $\mathbf{f}$ in Eq. (45) are obtained in the standard FEM fashion by assembling the element contribution given by

$$
\begin{aligned}
\mathbf{K}_{i j}^{e} & =\int_{\Omega^{e}}\left(\nabla^{T} N_{i}\right) \mathbf{D}_{T} \nabla N_{j} d \Omega-\int_{\Omega^{e}} \frac{1}{2}\left(\nabla^{T} N_{i}\right) \mathbf{h} \nabla^{T}\left(\mathbf{D} \nabla N_{j}\right) d \Omega \\
\mathbf{C}_{i j}^{e} & =-\int_{\Omega^{e}}\left(\nabla^{T} N_{i}\right) \overline{\mathbf{v}} N_{j} d \Omega \\
\mathbf{S}_{i j}^{e} & =\int_{\Omega^{e}} s N_{i} N_{j} d \Omega \\
\mathbf{f}_{i}^{e} & =\int_{\Omega^{e}}\left(N_{i}+\frac{1}{2}\left(\nabla^{T} N_{i}\right) \mathbf{h}\right) Q d \Omega-\oint_{\Gamma_{q}^{e}} N_{i} q d \Gamma
\end{aligned}
$$

Note that the second integral in Eq. (46) vanishes for linear finite element approximations.

\subsection{Particularization of the multidimensional formulation to the $1 D$ case}

The following simplifications in the FIC-FEM approach presented in Section 3.3 apply for the 1D case

$$
\mathbf{v}=\{v\} \quad, \quad \hat{\mathbf{v}}=\{1\} \quad, \quad \mathbf{D}=\{k\} \quad, \quad \mathbf{D}_{s}=\{0\} \quad, \quad \mathbf{h}=\{h\} \quad, \quad \mathbf{h}_{s_{c}}=\{0\}
$$

Taking into account Eq. (50), the expression for the characteristic length of Eq. (20) simplifies to the form of Eq. (14), i.e.

$$
h=h_{v}+h_{r}
$$

with $h_{v}=\alpha_{v} l^{e}$ and $h_{r}=2 \alpha_{r} \frac{k}{r_{s}} \frac{d \phi}{d x}$.

The expression of the stabilization parameter $\alpha_{v}$ of Eq. (26) coincides precisely with that given in Eq. (19a) simply noting that $\gamma_{v}=\gamma=\frac{v l^{e}}{2 k}$ and $w_{v}=w=\frac{s\left(l^{e}\right)^{2}}{k}$.

On the other hand, the expression for $\alpha_{r}$ is deduced from Eq. (28) making $\varphi=3, \gamma_{v}=\gamma$ and $\mathbf{D}_{s}=\{0\}$. This gives

$$
\alpha_{r}=\gamma\left[\frac{\sigma}{3}\left(\frac{\xi+2}{\xi-1}\right)-\alpha_{v}\right]-1
$$

which coincides with the 1D expression (Eq. (19b)). As mentioned earlier, both 1D expressions of $\alpha_{v}$ and $\alpha_{r}$ lead to exact nodal solutions for uniform meshes of 2-noded linear elements [48].

\section{Examples}

\subsection{Examples}

In this section we present the solutions obtained by the proposed FIC-FEM method for some stationary benchmark examples in 2D. The domain is $(x, y)=[0,1] \times[0,1]$. Each example is solved using the six different meshes shown in Fig. 1. The simplicial meshes consist of $2 \times 20 \times 20 \mathrm{P} 1$ finite elements and the quadrilateral meshes consist of $20 \times 20$ Q1 finite elements. The mesh node positions in Figs. 1(b), 1(c), 1(e) and 1(f) are obtained by randomly perturbing uniformly spaced nodes. The nodes adjacent to the domain boundary in Figs. 1(c) and 1(f) are perturbed only in the direction parallel to the boundary. The simplicial meshes in Figs. 1(b) and 1(c) are obtained by Delaunay tessellation. These meshes are called unstructured as every interior node has a distinct mesh topology in its neighbourhood. The meshes in Figs. 1(e) and 1(f) are obtained by adopting the connectivity of the uniform mesh in Fig. 1(d). In these meshes there are always four elements in the patch surrounding any interior node. As only the shapes of the elements vary inside a patch, these meshes are better denoted as nonuniform.

The solutions to the numerical examples are presented as surface plots whose view is described as $\left(\theta^{\circ}, \psi^{\circ}\right)$, where $\theta^{\circ}$ is the azimuthal angle with respect to the negative $y$-axis and $\psi^{\circ}$ is the elevation angle from the $x-y$ plane.

Example 1. The problem data is: $\mathbf{v}=\left[5 \cdot 10^{6},-9 \cdot 10^{6}\right]^{\mathrm{T}}, k_{1}=1, k_{2}=1, s=0$ and $Q=0$. The boundary conditions are: $\phi=1$ on $(x=0, y>0.7) \cup(x<1, y=1), \phi=0.5$ at $(x=0, y=0.7)$ and $\phi=0$ on the rest of the boundary. This problem was first introduced in [4]. The solution develops an exponential boundary layer 


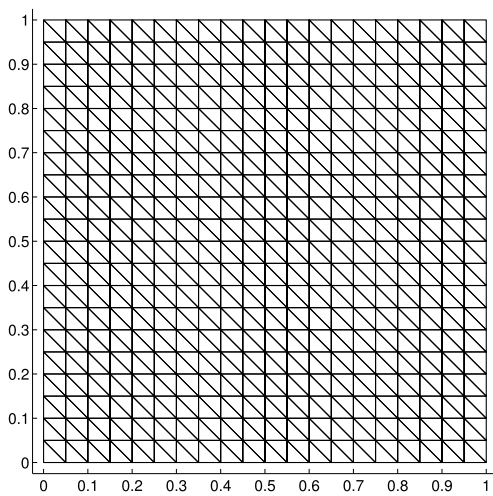

(a) P1 elements, structured.

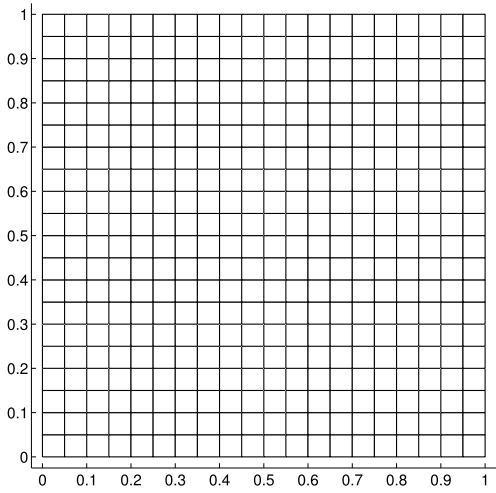

(d) Q1 elements, structured.

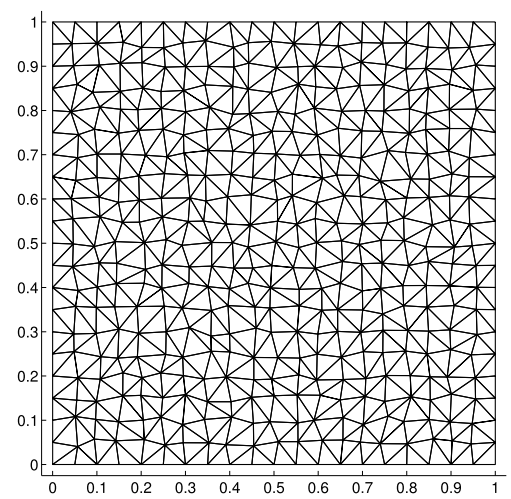

(b) P1 elements, unstructured-1.

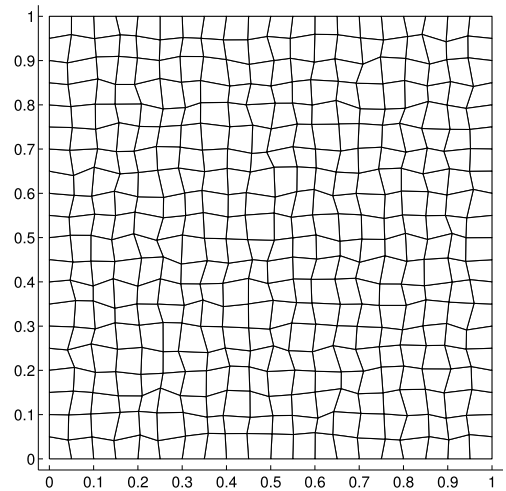

(e) Q1 elements, nonuniform-1.

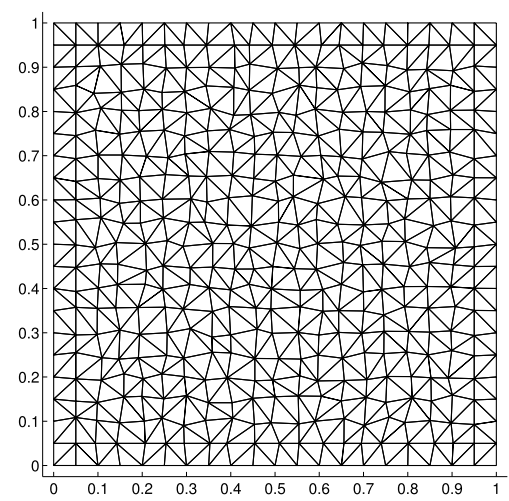

(c) P1 elements, unstructured-2.

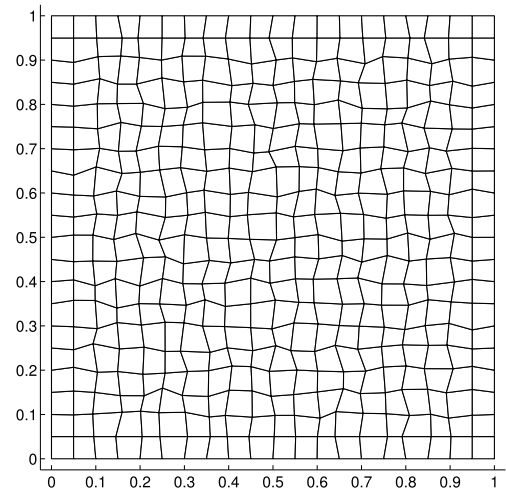

(f) Q1 elements, nonuniform-2.

Fig. 1. Simplicial (P1 elements) and quadrilateral (Q1 elements) meshes considered in the numerical examples. The nodes adjacent to the domain boundary in (c) and (f) are perturbed only in the direction parallel to the boundary.

at the outflow boundary and an internal characteristic layer (parabolic) which is skewed to the mesh and boundary. Both layers are subgrid phenomena for the considered mesh resolution. The FIC-FEM solutions are shown in Fig. 2 and are viewed at $\left(20^{\circ}, 20^{\circ}\right)$. Note that both the exponential and characteristic layers are reproduced in the FICFEM solution without spurious oscillations about the layers. Clearly, the best solution are obtained for the structured meshes.

Example 2. This is a uniform advection problem with a constant source term introduced in [65]. The problem data is: $\mathbf{v}=[1,0]^{\mathrm{T}}, k_{1}=10^{-8}, k_{2}=10^{-8}, s=0$ and $Q=1$. The homogeneous boundary condition $\phi=0$ is imposed everywhere. The solution develops an exponential layer at the outflow boundary $x=1$ and parabolic layers at the boundaries $y=0$ and $y=1$. Both layers are subgrid phenomena for the considered mesh resolution. The FIC-FEM solutions are shown in Fig. 3 and are viewed at $\left(-45^{\circ}, 20^{\circ}\right)$. Again the best solutions are obtained for the structural meshes.

Example 3. This is a uniform advection problem with a discontinuous source term introduced in [29]. The problem data is: $\mathbf{v}=[1,0]^{\mathrm{T}}, k_{1}=10^{-8}, k_{2}=10^{-8}, s=0, Q(x<0.5)=1$ and $Q(x>0.5)=-1$. The homogeneous boundary condition $\phi=0$ is imposed everywhere. Visually the solution looks like a prism of height 0.5 with the kink positioned at $x=0.5$. However, the solution develops parabolic layers at the boundaries $y=0$ and $y=1$. Further, it also develops exponential layers near the corners of the boundary $x=1$ where the parabolic layers meet. Both layers are subgrid phenomena for the considered mesh resolution. The Galerkin FEM will yield a solution which exhibits localized Gibbs oscillations across the parabolic layers and spurious global oscillations along the parabolic layers. 


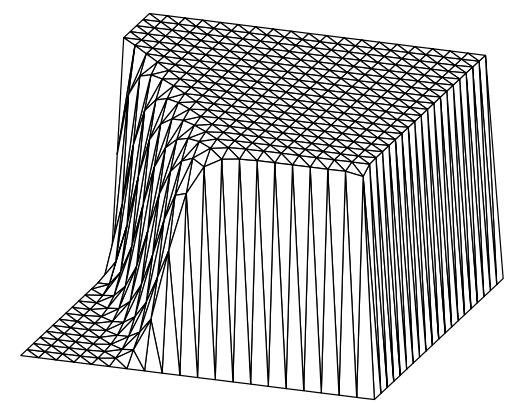

(a) P1 elements, structured.

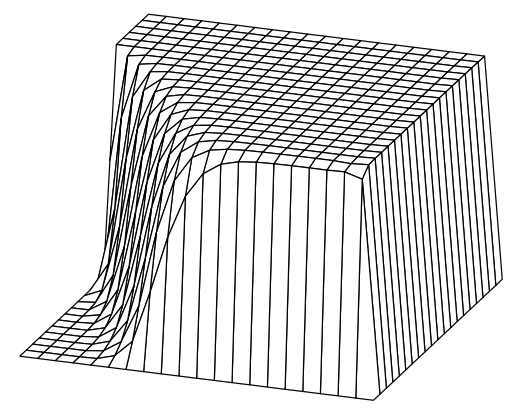

(d) Q1 elements, structured.

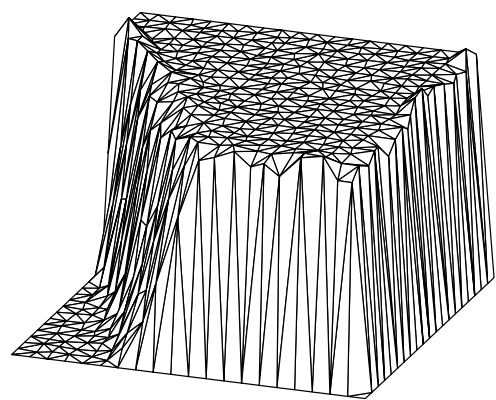

(b) P1 elements, unstructured-1.

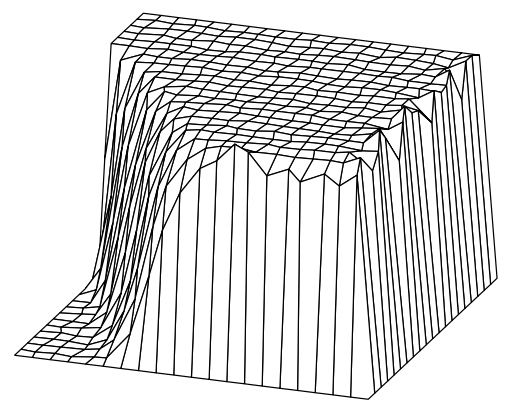

(e) Q1 elements, nonuniform-1.

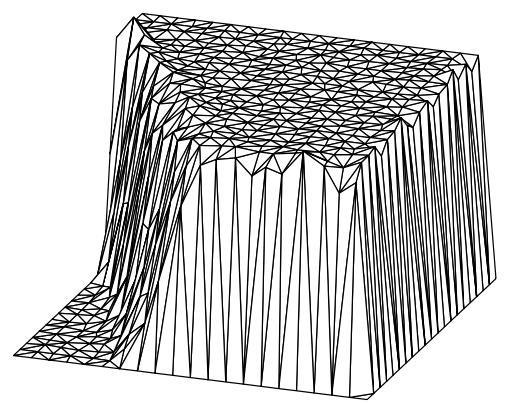

(c) P1 elements, unstructured-2.

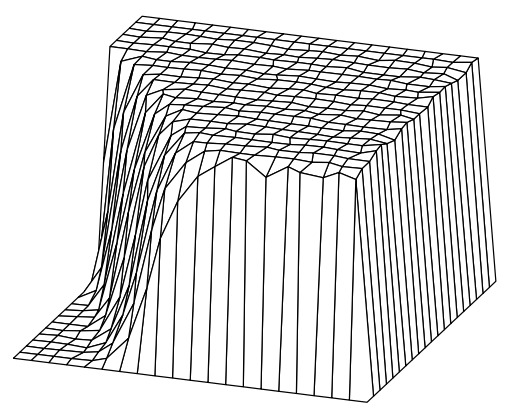

(f) Q1 elements, nonuniform-2.

Fig. 2. FIC-FEM solution to Example 1. $\mathbf{v}=\left[5 \cdot 10^{6},-9 \cdot 10^{6}\right]^{\mathrm{T}}, k_{1}=1, k_{2}=1, s=0, Q=0$ and viewed at $\left(20^{\circ}, 20^{\circ}\right)$.

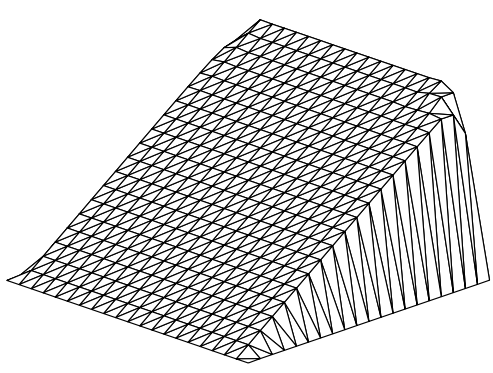

(a) P1 elements, structured.

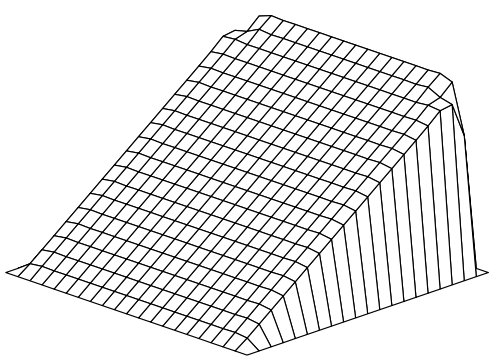

(d) Q1 elements, structured.

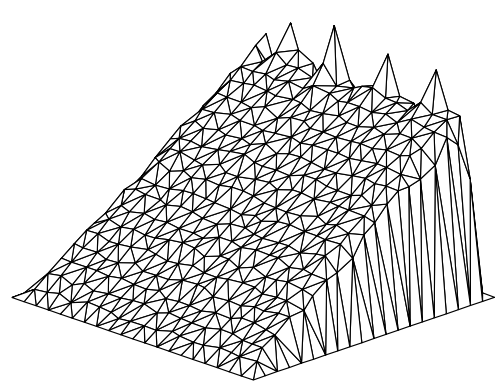

(b) P1 elements, unstructured-1.

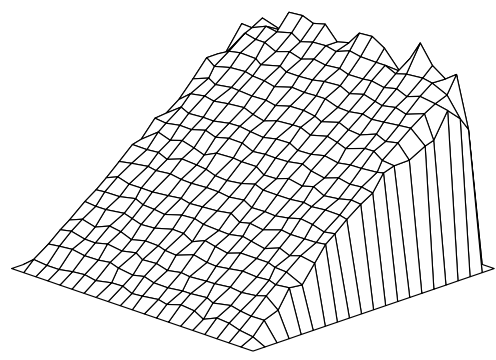

(e) Q1 elements, nonuniform-1.

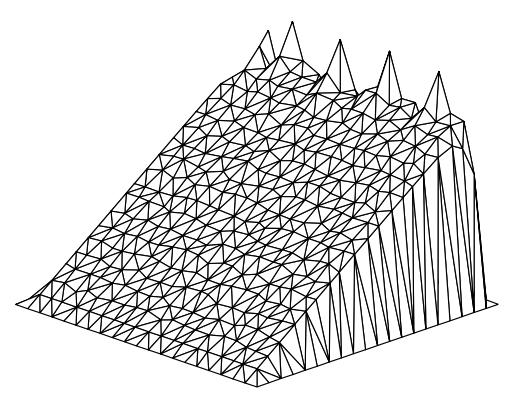

(c) P1 elements, unstructured-2.

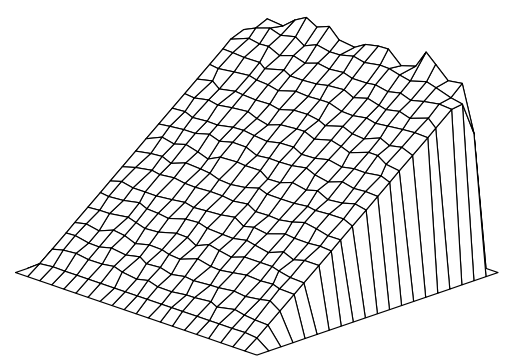

(f) Q1 elements, nonuniform-2.

Fig. 3. FIC-FEM solution to Example 2. $\mathbf{v}=[1,0]^{\mathrm{T}}, k_{1}=10^{-8}, k_{2}=10^{-8}, s=0, Q=1$ and viewed at $\left(-45^{\circ}, 20^{\circ}\right)$.

The presence of the exponential layer in the neighbourhood of the corners (although invisible to the naked eye) is the cause of the spurious global oscillations. The FIC-FEM solutions are shown in Fig. 4 and are viewed at $\left(-10^{\circ}, 20^{\circ}\right)$. 


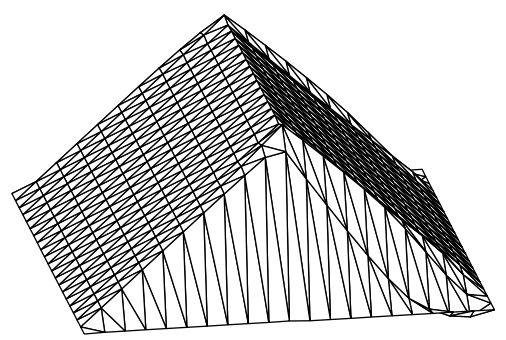

(a) P1 elements, structured.

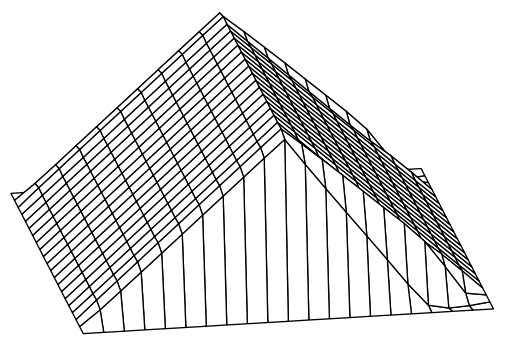

(d) Q1 elements, structured.

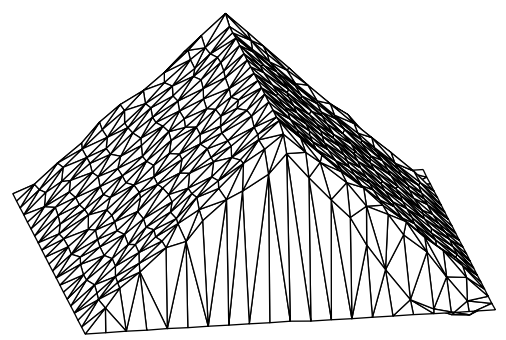

(b) P1 elements, unstructured-1.

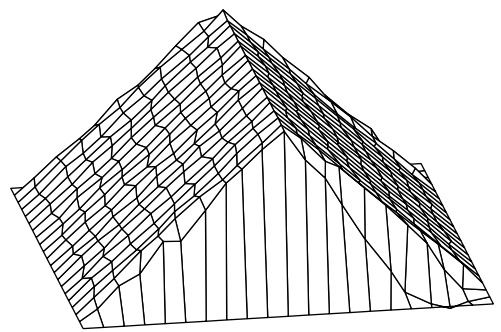

(e) Q1 elements, nonuniform-1.

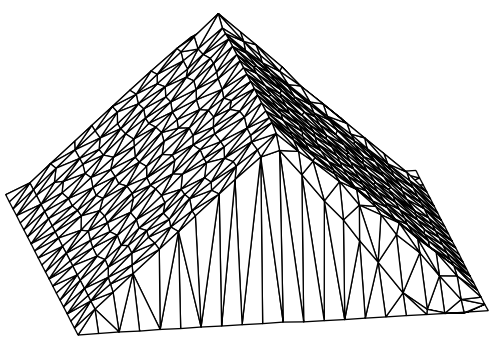

(c) P1 elements, unstructured-2.

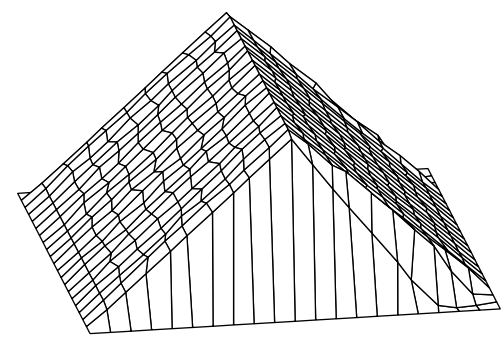

(f) Q1 elements, nonuniform-2.

Fig. 4. The FIC-FEM solution to Example 3. $\mathbf{v}=[1,0]^{\mathrm{T}}, k_{1}=10^{-8}, k_{2}=10^{-8}, s=0, Q(x<0.5)=1, Q(x>0.5)=-1$ and viewed at $\left(-10^{\circ}, 20^{\circ}\right)$.

This problem also emphasizes the necessity of a consistent stabilization method: the position and height of the kink found in the solution at $x=0.5$ will be incorrectly predicted otherwise. Note the higher accuracy obtained for the structural meshes.

Example 4. This is a plain diffusion-absorption problem. The problem data is: $\mathbf{v}=\mathbf{0}, k_{1}=10^{-4}, k_{2}=10^{-8}, s=1$ and $Q=1$. The homogeneous boundary condition $\phi=0$ is imposed everywhere. As there is no advection velocity, the solution only develops parabolic layers at the boundary. Due to a larger diffusion coefficient along the $x$-axis, the layers at $x=0$ and $x=1$ are resolved by the mesh. The layers at $y=0$ and $y=1$ are subgrid phenomena for the considered mesh resolution. The FIC-FEM solutions are shown in Fig. 5 and are viewed at $\left(-45^{\circ}, 20^{\circ}\right)$.

Example 5. This is a convection-diffusion-absorption problem and is a multidimensional modification of the 1D problem studied earlier in [20,46]. The problem data is: $\mathbf{v}=\left[10^{-2}, 0\right]^{\mathrm{T}}, k_{1}=10^{-4}, k_{2}=10^{-4}, s=4.8$ and $Q=0$. The boundary conditions are: $\phi=1.0$ on $(x=0, y) \cup(x, y=0), \phi=(3 / 8)$ on the rest of the boundary. The solution develops a weak exponential layer at the boundary $x=1$ and parabolic layers at the remaining boundaries. The distinctive feature of this problem is that Gibbs oscillations found at the upwind boundary $x=0$ in the solution of Galerkin FEM were found to be enhanced (instead of diminished; cf. [46, Section 5.7.1], [47]) in the solutions obtained using the SUPG method [4], the ASGS method [66] and the CAU method [23]. In the latter methods, the expression multiplying the reaction coefficient in the stabilization terms introduces a negative advection effect which causes this abnormal behaviour. The FIC-FEM solutions presented in [20,47] and the solution of the SGS-GSGS method [42] successfully control the numerical oscillations for this problem. The solutions obtained by the proposed FIC-FEM method viewed at $\left(120^{\circ}, 20^{\circ}\right)$ are shown in Fig. 6. Stabilized and accurate results are obtained in all cases.

Example 6. This problem is similar to the one considered in Example 5 except for the following changes. The magnitude of the advection velocity is increased to $\mathbf{v}=[1,0]^{\mathrm{T}}$ and of the diffusion coefficients are reduced to $k_{1}=10^{-8}, k_{2}=10^{-8}$. The increased advection strengthens the exponential layer at $x=1$ and smears the parabolic layer at $x=0$ by sweeping the imposed boundary condition into the domain. The reduced diffusion strengthens the parabolic layers at $y=0$ and $y=1$. Both layers are subgrid phenomena for the considered mesh resolution. The 


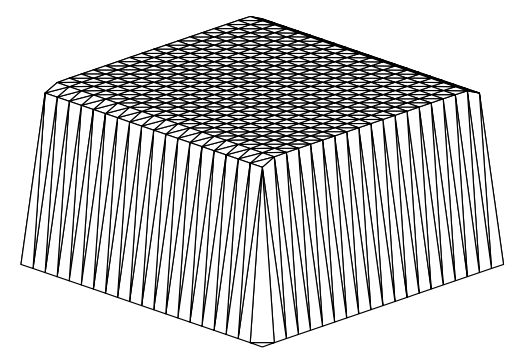

(a) P1 elements, structured.

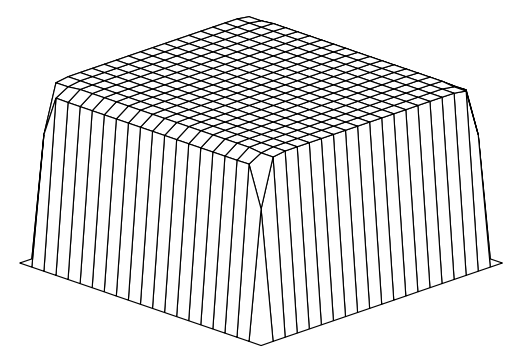

(d) Q1 elements, structured.

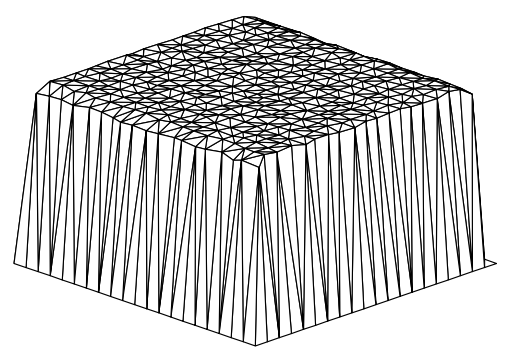

(b) P1 elements, unstructured-1.

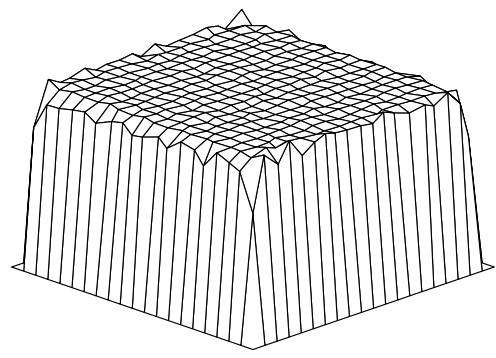

(e) Q1 elements, nonuniform-1.

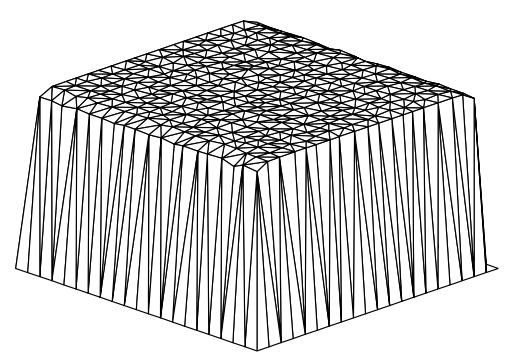

(c) P1 elements, unstructured-2.

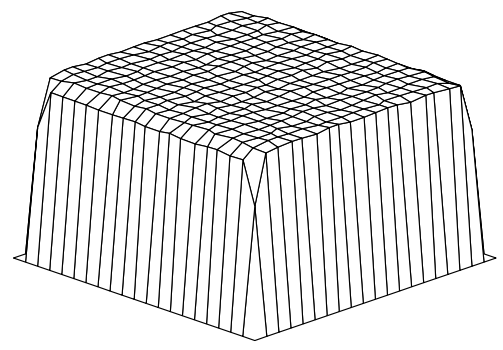

(f) Q1 elements, nonuniform-2.

Fig. 5. The FIC-FEM solution to Example 4. $\mathbf{v}=\mathbf{0}, k_{1}=10^{-4}, k_{2}=10^{-8}, s=1, Q=1$ and viewed at $\left(-45^{\circ}, 20^{\circ}\right)$.

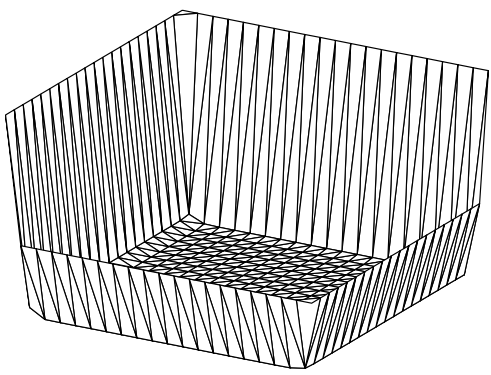

(a) P1 elements, structured.

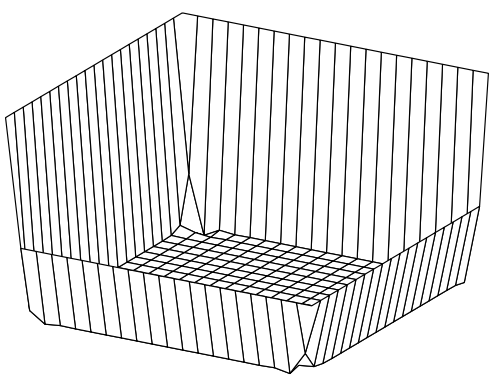

(d) Q1 elements, structured.

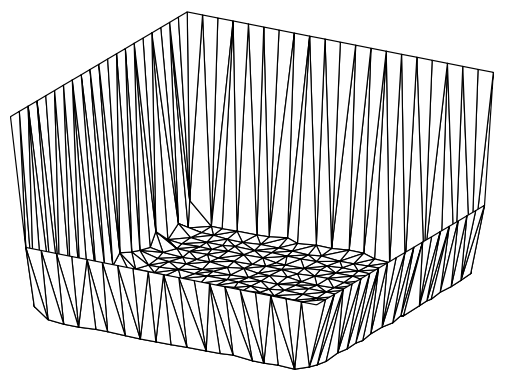

(b) P1 elements, unstructured-1.

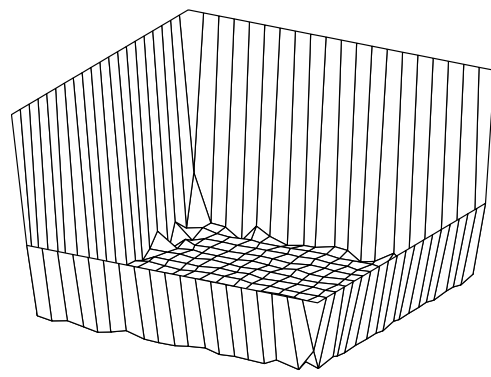

(e) Q1 elements, nonuniform-1.

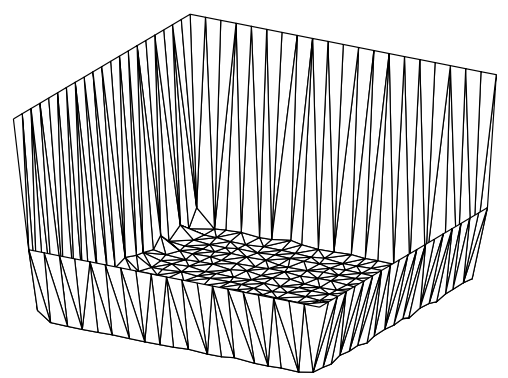

(c) P1 elements, unstructured-2.

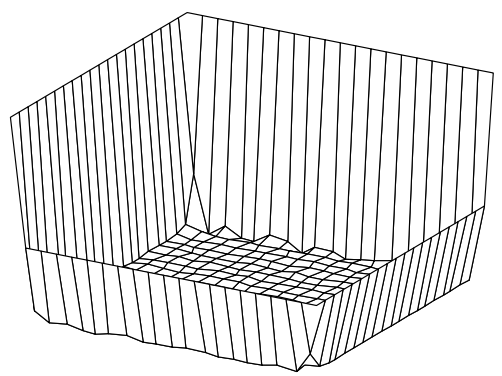

(f) Q1 elements, nonuniform-2.

Fig. 6. The FIC-FEM solution to Example 5. $\mathbf{v}=\left[10^{-2}, 0\right]^{\mathrm{T}}, k_{1}=10^{-4}, k_{2}=10^{-4}, s=4.8, Q=0$ and viewed at $\left(120^{\circ}, 20^{\circ}\right)$.

FIC-FEM solutions viewed at $\left(120^{\circ}, 20^{\circ}\right)$ are shown in Fig. 7. Solutions are again stable and accurate. Once more the best results are obtained using structured meshes. 


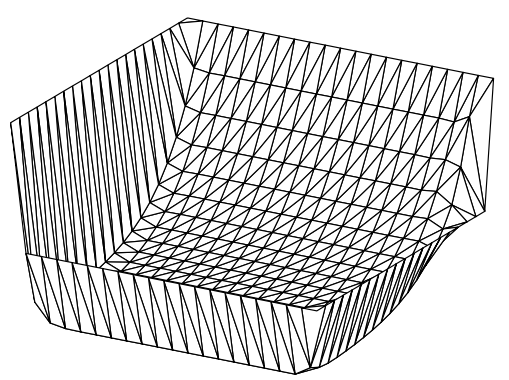

(a) P1 elements, structured.

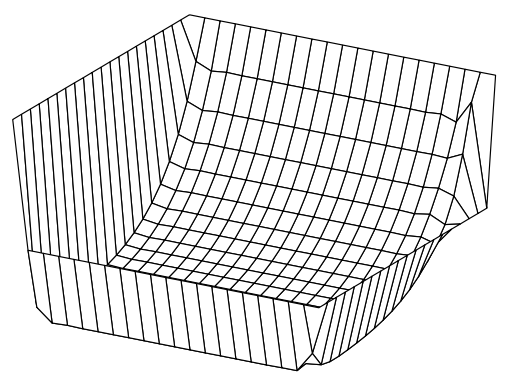

(d) Q1 elements, structured.

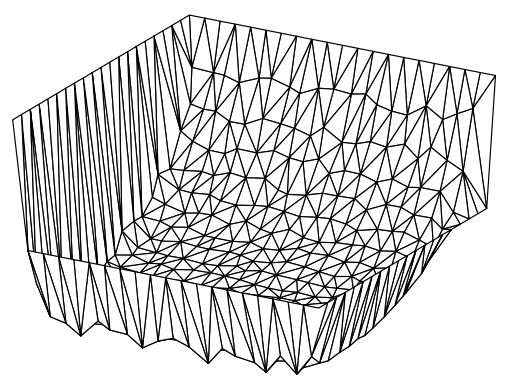

(b) P1 elements, unstructured-1.

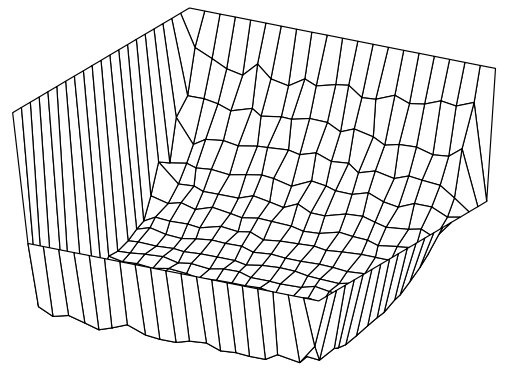

(e) Q1 elements, nonuniform-1.

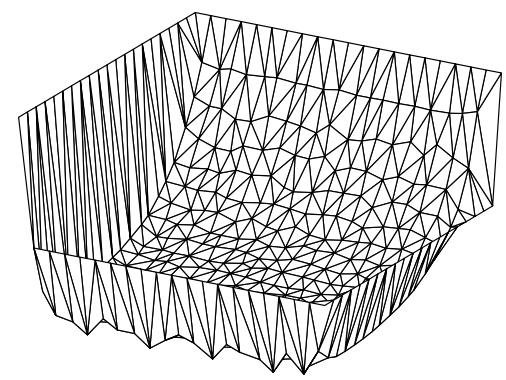

(c) P1 elements, unstructured-2.

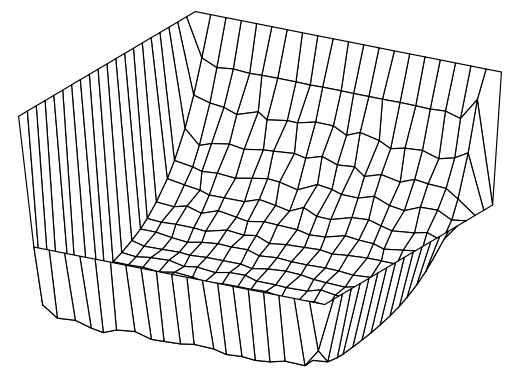

(f) Q1 elements, nonuniform-2.

Fig. 7. The FIC-FEM solution to Example 6. $\mathbf{v}=[1,0]^{\mathrm{T}}, k_{1}=10^{-8}, k_{2}=10^{-8}, s=4.8, Q=0$ and viewed at $\left(120^{\circ}, 20^{\circ}\right)$.

\section{Concluding remarks}

The two-parameters stabilized FIC-FEM formulation presented has proven to give accurate results for a range of 2D steady-state advection-diffusion-absorption problems. The extension of the formulation to transient problems is possible follows the ideas presented in [48]. This extension will be reported in a subsequent work.

\section{Acknowledgements}

This research was partially funded by the ICEBREAKER project of the European Research Council. Support for this work was also provided from the Office for Naval Research Global (ONRG) of the US Navy through the NICESHIP project (Award N62909-16-1-2236). We also acknowledge the financial support of the CERCA programme of the Generalitat de Catalunya.

\section{References}

[1] J. Donea, A. Huerta, Finite Element Method for Flow Problems, J. Wiley \& Sons, 2003.

[2] O.C. Zienkiewicz, R.L. Taylor, J.Z. Zhu, The Finite Element Method. The Basis, sixth ed., Elsevier, 2005.

[3] O.C. Zienkiewicz, R.L. Taylor, P. Nithiarasu, The Finite Element Method for Fluid Dynamics, sixth ed., Elsevier, 2005.

[4] A.N. Brooks, T.J.R. Hughes, Streamline upwind/Petrov-Galerkin formulations for the convective dominated flows with particular emphasis on the incompressible Navier-Stokes equations, Comput. Methods Appl. Mech. Engrg. 32 (1-3) (1982) 199-259.

[5] T.J.R. Hughes, A.N. Brooks, A theoretical framework for Petrov-Galerkin methods, with discontinuous weighting functions: application to the streamline upwind procedure, in: R.H. Gallagher, D.M. Norrie, J.T. Oden, Zienkiewicz O.C. (Eds.), Finite Elements in Fluids, IV,Wiley, Chichester, 1982.

[6] T.J.R. Hughes, M. Mallet, A new finite element formulations for computational fluid dynamics: III. The generalized streamline operator for multidimensional advective-diffusive systems, Comput. Methods Appl. Mech. Engrg. 58 (1986) 305-328.

[7] J. Donea, A Taylor-Galerkin method for convective transport problems, Internat. J. Numer. Methods Engrg. 20 (1984) $101-119$.

[8] J. Douglas, T.F. Russell, Numerical methods for convection-dominated diffusion problems based on combining the method of characteristics with the finite element or finite difference procedures, SIAM J. Numer. Anal. 19 (1982) 871-885.

[9] R. Löhner, K. Morgan, O.C. Zienkiewicz, The solution of non-linear hyperbolic equation systems by the finite element method, Internat. J. Numer. Methods Fluids 4 (1984) 1043-1063. 
[10] T.J.R. Hughes, L.P. Franca, G.M. Hulbert, A new finite element formulation for computational fluid dynamics: VIII. The Galerkin/leastsquares method for advective-diffusive equations, Comput. Methods Appl. Mech. Engrg. 73 (1989) 173-189.

[11] F. Brezzi, M.O. Bristeau, L.P. Franca, M. Mallet, G. Rogé, A relationship between stabilized finite element methods and the Galerkin method with bubble functions, Comput. Methods Appl. Mech. Engrg. 96 (1992) 117-129.

[12] C. Baiocchi, F. Brezzi, L.P. Franca, Virtual bubbles and the GaLS, Comput. Methods Appl. Mech. Engrg. 105 (1993) $125-141$.

[13] F. Brezzi, A. Russo, Choosing bubbles for advection-diffusion problems, Math. Models Methods Appl. Sci. 4 (1994) $571-587$.

[14] T.J.R. Hughes, G.R. Feijoo, L. Mazzei, J.B. Quincy, The variational multiscale method: a paradigm for computational mechanics, Comput. Methods Appl. Mech. Engrg. 166 (1998) 3-24.

[15] O.C. Zienkiewicz, R. Codina, A general algorithm for compressible and incompressible flows. Part I: the split, characteristic based scheme, Internat. J. Numer. Methods Fluids 20 (1995) 869-885.

[16] E. Oñate, Derivation of stabilized equations for numerical solution of advective-diffusive transport and fluid flow problems, Comput. Methods Appl. Mech. Engrg. 151 (1998) 233-265.

[17] E. Oñate, M. Manzan, A general procedure for deriving stabilized space-time finite element methods for advective-diffusive problems, Internat. J. Numer. Methods Fluids 31 (1999) 203-221.

[18] E. Oñate, M. Manzan, Stabilization techniques for finite element analysis of convection-diffusion problems, in: B. Sundén, G. Comini (Eds.), Computational Analysis of Convection Heat Transfer, WIT Press, Southampton, UK, 2000, pp. 71-117.

[19] E. Oñate, F. Zarate, S.R. Idelsohn, Finite element formulation for the convective-diffusive problems with sharp gradients using finite calculus, Comput. Methods Appl. Mech. Engrg. 195 (2006) 1793-1825.

[20] E. Oñate, J. Miquel, G. Hauke, Stabilized formulation for the advection-diffusion-absorption equation using finite calculus and linear finite elements, Comput. Methods Appl. Mech. Engrg. 195 (33-36) (2006) 3926-3946.

[21] E. Oñate, J. Miquel, F. Zarate, Stabilized solution of the multidimensional advection-diffusion-absorption equation using linear finite elements, Comput. \& Fluids 36 (2007) 92-112.

[22] R. Codina, Comparison of some finite element methods for solving the diffusion-convection-reaction equation, Comput. Methods Appl. Mech. Engrg. 156 (1998) 185-210.

[23] A.C. Galeão, E.G. Dutra do Carmo, A consistent approximate upwind Petrov-Galerkin method for the convection-dominated problems, Comput. Methods Appl. Mech. Engrg. 68 (1) (1988) 83-95.

[24] R. Codina, A discontinuity-capturing crosswind-dissipation for the finite element solution of the convection-diffusion equation, Comput. Methods Appl. Mech. Engrg. 110 (1993) 325-342.

[25] P.A.B. de Sampaio, A.L.G.A. Coutinho, A natural derivation of discontinuity capturing operator for convection-diffusion problems, Comput. Methods Appl. Mech. Engrg. 190 (2001) 6291-6308.

[26] E.G. Dutra do Carmo, G.B. Alvarez, A new upwind function in stabilized finite element formulations, using linear and quadratic elements for the scalar convection-diffusion problems, Comput. Methods Appl. Mech. Engrg. 193 (2004) 2383-2402.

[27] C. Johnson, A. Szepessy, P. Hansbo, On the convergence of shock-capturing streamline diffusion finite element methods for hyperbolic conservation laws, Math. Comp. 54 (1990) 107-129.

[28] P. Knobloch, Improvements of the Mizukami-Hughes method for convection-diffusion equations, Comput. Methods Appl. Mech. Engrg. 196 (2006) 579-594.

[29] A. Mizukami, T.J.R. Hughes, A Petrov-Galerkin finite element method for convection-dominated flows: An accurate upwinding technique for satisfying the maximum principle, Comput. Methods Appl. Mech. Engrg. 50 (2) (1985) 181-193.

[30] V. John, P. Knobloch, On spurious oscillations at layers diminishing (SOLD) methods for convection-diffusion equations: Part I - A review, Comput. Methods Appl. Mech. Engrg. 196 (2007) 2197-2215.

[31] E. Burman, A. Ern, Nonlinear diffusion and discrete maximum principle for stabilized Galerkin approximations of the convection-diffusionreaction equation, Comput. Methods Appl. Mech. Engrg. 191 (2002) 3833-3855.

[32] T.E. Tezduyar, Y.J. Park, Discontinuity-capturing finite element formulations for nonlinear convection-diffusion-reaction equations, Comput. Methods Appl. Mech. Engrg. 59 (1986) 307-325.

[33] S.R. Idelsohn, N. Nigro, M. Storti, G. Buscaglia, A Petrov-Galerkin formulation for advective-reaction-diffusion problems, Comput. Methods Appl. Mech. Engrg. 136 (1996) 27-46.

[34] L.P. Franca, E.G. Dutra do Carmo, The Galerkin gradient least-squares method, Comput. Methods Appl. Mech. Engrg. 74 (1989) $41-54$.

[35] I. Harari, T.J.R. Hughes, Stabilized finite element methods for steady advection-diffusion with production, Comput. Methods Appl. Mech. Engrg. 115 (1994) 165-191.

[36] L.P. Franca, C. Farhat, Bubble functions prompt unusual stabilized finite element methods, Comput. Methods Appl. Mech. Engrg. 123 (1995) 299-308.

[37] L.P. Franca, F. Valentin, On an improved unusual stabilized finite element method for the advective-reactive-diffusive equation, Comput. Methods Appl. Mech. Engrg. 190 (2001) 1785-1800.

[38] F. Brezzi, G. Hauke, L.D. Marini, G. Sangalli, Link-cutting bubbles for the stabilization of convection-diffusion-reaction problems, Math. Models Methods Appl. Sci. 13 (3) (2003) 445-461.

[39] R. Codina, On stabilized finite element methods for linear systems of convection-diffusion-reaction equations, Comput. Methods Appl. Mech. Engrg. 19 (2006) 2681-2706.

[40] G. Hauke, A. García-Olivares, Variational subgrid scale formulations for the advection-diffusion-reaction equation, Comput. Methods Appl. Mech. Engrg. 19 (2001) 6847-6865.

[41] G. Hauke, A simple subgrid scale stabilized method for the advection-diffusion-reaction equation, Comput. Methods Appl. Mech. Engrg. 191 (2002) 2925-2947. 
[42] G. Hauke, G. Sangalli, M.H. Doweidar, Combining adjoint stabilized methods for the advection-diffusion-reaction problem, Math. Models Methods Appl. Sci. 17 (2) (2007) 305-326.

[43] C. Felippa, E. Oñate, Nodally exact Ritz discretizations of the 1D diffusion-absorption and Helmholtz equations by variational FIC and modified equation methods, Comput. Mech. 39 (2) (2007) 91-111.

[44] S.R. Idelsohn, J.C. Heinrich, E. Oñate, Petrov-Galerkin methods for the transient advective-diffusive equation with sharp gradients, Internat. J. Numer. Methods Engrg. 39 (1996) 1455-1473.

[45] C.C. Yu, J.C. Heinrich, Petrov-Galerkin methods for the time-dependent convective transport equations, Internat. J. Numer. Methods Engrg. 23 (1986) 883-901.

[46] P. Nadukandi, E. Oñate, J. Espinosa García, A high-resolution Petrov-Galerkin method for the 1D convection-diffusion-reaction problem, Comput. Methods Appl. Mech. Engrg. 199 (9-12) (2010) 525-546.

[47] P. Nadukandi, E. Oñate, J. Espinosa García, A high-resolution Petrov-Galerkin method for the convection-diffusion-reaction problem. Part II. A multidimensional extension, Comput. Methods Appl. Mech. Engrg. 213-216 (2012) 327-352.

[48] E. Oñate, J. Miquel, P. Nadukandi, An accurate FIC-FEM formulation for the 1D advection-diffusion-reaction equation, Comput. Methods Appl. Mech. Engrg. 298 (2016) 373-406.

[49] E. Oñate, A. Valls, J. García, FIC/FEM formulation with matrix stabilizing terms for incompressible flows at low and high Reynolds numbers, Comput. Mech. 38 (4-5) (2006) 440-455.

[50] E. Oñate, J. García, S.R. Idelsohn, F. Del Pin, FIC formulations for finite element analysis of incompressible flows. Eulerian, ALE and Lagrangian approaches, Comput. Methods Appl. Mech. Engrg. 195 (23-24) (2006) 3001-3037.

[51] E. Oñate, A. Valls, J. García, Modeling incompressible flows at low and high Reynolds numbers via a finite calculus-finite element approach, J. Comput. Phys. 224 (2007) 332-351.

[52] E. Oñate, P. Nadukandi, S. Idelsohn, J. García, C. Felippa, A family of residual-based stabilized finite element methods for stokes flows, Internat. J. Numer. Methods Fluids 65 (1-3) (2011) 106-134.

[53] E. Oñate, P. Nadukandi, S. Idelsohn, P1/P0+ elements for incompressible flows with discontinuous material properties, Comput. Methods Appl. Mech. Engrg. 271 (2014) 185-209.

[54] E. Oñate, J. García, A finite element method for fluid-structure interaction with surface waves using a finite calculus formulation, Comput. Methods Appl. Mech. Engrg. 191 (2001) 635-660.

[55] E. Oñate, S.R. Idelsohn, F. Del Pin, R. Aubry, The particle finite element method. An overview, Int. J. Comput. Methods 1 (2) (2004) $267-307$.

[56] E. Oñate, S.R. Idelsohn, M.A. Celigueta, R. Rossi, Advances in the particle finite element method for the analysis of fluid-multibody interaction and bed erosion in free surface flows, Comput. Methods Appl. Mech. Engrg. 197 (19-20) (2008) 1777.

[57] E. Oñate, R.L. Taylor, O.C. Zienkiewicz, J. Rojek, A residual correction method based on finite calculus, Eng. Comput. 20 (5/6) (2003) 629-658.

[58] E. Oñate, J. Rojek, O.C. Zienkiewicz, R.L. Taylor, Finite calculus formulation for incompressible solids using linear triangles and tetrahedra, Internat. J. Numer. Methods Engrg. 59 (2004) 1473-1500.

[59] E. Oñate, S.R. Idelsohn, C. Felippa, Consistent pressure Laplacian stabilization for incompressible continua via higher order finite calculus, Internat. J. Numer. Methods Engrg. 87 (1-5) (2011) 171-195.

[60] E. Oñate, S.R. Idelsohn, O.C. Zienkiewicz, R.L. Taylor, C. Sacco, A stabilized finite point method for analysis of fluid mechanics problems, Comput. Methods Appl. Mech. Engrg. 139 (1996) 315-346.

[61] E. Oñate, S.R. Idelsohn, A mesh-free finite point method for advective-diffusive transport and fluid flow problems, Comput. Mech. 23 (1998) 283-292.

[62] E. Oñate, C. Sacco, S.R. Idelsohn, A finite point method for incompressible flow problems, Comput. Vis. Sci. 2 (2000) 67-75.

[63] E. Oñate, F. Perazzo, J. Miquel, A finite point method for elasticity problems, Comput. Struct. 79 (2001) 2151-2163.

[64] E. Oñate, Possibilities of finite calculus in computational mechanics, Internat. J. Numer. Methods Engrg. 60 (1) (2004) $255-281$.

[65] F. Kikuchi, T. Ushijima, Theoretical analysis of some finite element schemes for convective diffusion equations, in: R. Gallagher, D. Norrie, J. Oden, O.C. Zienkiewicz (Eds.), Finite Elements in Fluids, Vol. IV, John Wiley and Sons Ltd, Chichester, 1982.

[66] J. Principe, R. Codina, On the stabilization parameter in the subgrid scale approximation of scalar convection-diffusion-reaction equations on distorted meshes, Comput. Methods Appl. Mech. Engrg. 199 (2122) (2010) 1386-1402. 